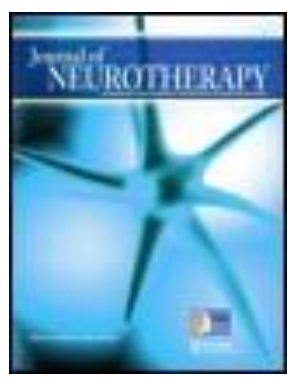

Journal of Neurotherapy: Investigations in Neuromodulation, Neurofeedback and Applied Neuroscience

\title{
LORETA Neurofeedback for Addiction and the Possible Neurophysiology of Psychological Processes Influenced: A Case Study and Region of Interest Analysis of LORETA Neurofeedback in Right Anterior Cingulate Cortex
}

\author{
Rex Cannon $M A^{a}$, Joel Lubar PhD ${ }^{a}$, Estate Sokhadze PhD ${ }^{b} \&$ Debora Baldwin PhD ${ }^{a}$ \\ a Department of Psychology, University of Tennessee , \\ b Department of Psychiatry and Behavioral Sciences, University of Louisville , \\ Published online: 12 Dec 2008.
}

To cite this article: Rex Cannon MA, Joel Lubar PhD, Estate Sokhadze PhD \& Debora Baldwin PhD (2008) LORETA Neurofeedback for Addiction and the Possible Neurophysiology of Psychological Processes Influenced: A Case Study and Region of Interest Analysis of LORETA Neurofeedback in Right Anterior Cingulate Cortex, Journal of Neurotherapy: Investigations in Neuromodulation, Neurofeedback and Applied Neuroscience, 12:4, 227-241, DOI: 10.1080/10874200802501948

To link to this article: http://dx.doi.org/10.1080/10874200802501948

PLEASE SCROLL DOWN FOR ARTICLE

( ) International Society for Neurofeedback and Research (ISNR), all rights reserved. This article (the "Article") may be accessed online from ISNR at no charge. The Article may be viewed online, stored in electronic or physical form, or archived for research, teaching, and private study purposes. The Article may be archived in public libraries or university libraries at the direction of said public library or university library. Any other reproduction of the Article for redistribution, sale, resale, loan, sublicensing, systematic supply, or other distribution, including both physical and electronic reproduction for such purposes, is expressly forbidden. Preparing or reproducing derivative works of this article is expressly forbidden. ISNR makes no representation or warranty as to the accuracy or completeness of any content in the Article. From 1995 to 2013 the Journal of Neurotherapy was the official publication of ISNR (www. Isnr.org); on April 27, 2016 ISNR acquired the journal from Taylor \& Francis Group, LLC. In 2014, ISNR established its official open-access journal NeuroRegulation (ISSN: 2373-0587; www. neuroregulation.org).

THIS OPEN-ACCESS CONTENT MADE POSSIBLE BY THESE GENEROUS SPONSORS

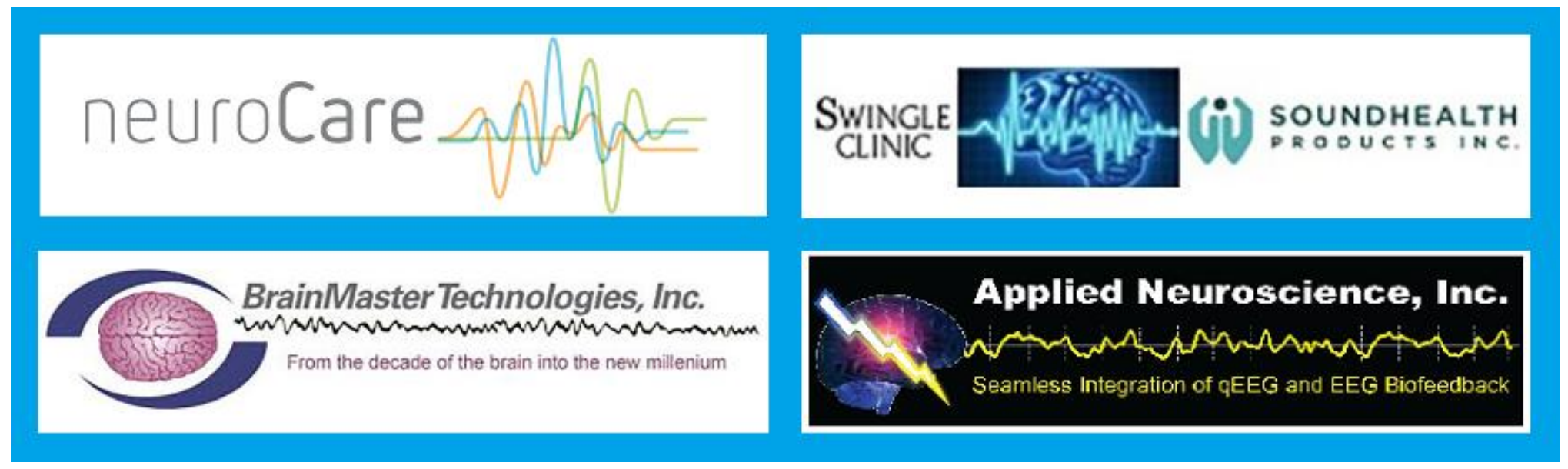




\title{
LORETA Neurofeedback for Addiction and the Possible Neurophysiology of Psychological Processes Influenced: A Case Study and Region of Interest Analysis of LORETA Neurofeedback in Right Anterior Cingulate Cortex
}

\author{
Rex Cannon, MA \\ Joel Lubar, PhD \\ Estate Sokhadze, PhD \\ Debora Baldwin, PhD
}

\begin{abstract}
Introduction. This case study explores the efficacy of low-resolution electromagnetic tomographic (LORETA) neurofeedback (LNFB) in the right anterior cingulate cortex (ACC) as a method for addiction treatment and examines the frequency specific effects of this training in eight other regions of the cortex identified as playing an important role in substance use disorders.

Methods. This case study was completed with one right-handed, 28-year-old female participant with 3 years of continuous abstinence from polysubstance abuse; her drugs of choice were heroin and alcohol, and she reported an 8-year history of alcohol abuse and a 4-year history of heroin use (IV). She completed 25 sessions of LNFB training in which she increased $14-18 \mathrm{~Hz}$ activity in the right ACC. We utilized electrophysiological measures to assess the increase or decrease in eight regions of interest (ROI): the right hippocampus, the right amygdaloid complex, the right orbitofrontal cortex (OFC), the right occipital lobe, the right insular cortex, the right uncus, and two regions in the left prefrontal cortex and compared them using complex linear mixed model and partial correlation procedures.

Results. The data indicate significant associations between these limbic and cortical regions. The linear increase in the right ACC was in the desired direction; however, this will require more than 25 sessions to reach significance. The effects of training in the right ACC show significant increase and decrease for all frequencies in specific regions of interest.

Conclusion. This is the first study of its kind to explore the relationship between these nine ROI as influenced by LNFB in the right ACC. The data suggest that these regions may play
\end{abstract}

Rex Cannon is affiliated with the Department of Psychology, University of Tennessee.

Joel Lubar is affiliated with the Department of Psychology, University of Tennessee.

Estate Sokhadze is affiliated with the Department of Psychiatry and Behavioral Sciences, University of Louisville.

Debora Baldwin is affiliated with the Department of Psychology, University of Tennessee.

Address correspondence to: Rex Cannon, MA, Department of Psychology, Suite 312, Austin Peay Building, University of Tennessee, Knoxville, TN 37921 (E-mail: rcannon2@utk.edu).

Journal of Neurotherapy, Vol. 12(4) 2008

Copyright ( 2008 ISNR. All rights reserved. doi: $10.1080 / 10874200802501948$ 
an intricate role in behaviors and characteristics involved in addiction; specific changes in the alpha frequency in limbic regions and increases in associations between regions in the theta frequency may influence personality and other behaviors associated with addictive traits. This case study illustrates the possible neural mechanisms involved in the negative self-reference associated with addiction even after a significant period of abstinence and possibly offers insight into antecedents to the onset of substance use disorders.

KEYWORDS. Addiction, addiction treatment, amygdala, anterior cingulate, EEG biofeedback, LORETA neurofeedback, neurofeedback

\section{INTRODUCTION}

Substance use disorders (SUDs) continue to exact innumerable costs on society and addicted individuals alike; unfortunately, in many instances available treatments for alcohol and drug dependence are considered inadequate (Blum et al., 2007; Köhnke et al., 2003). Electroencephalography (EEG) biofeedback (referred to also as neurofeedback, or neurotherapy) techniques have been utilized in clinical and research settings for treatment of neurological and psychiatric disorders (Lubar, 1997; Lubar \& Lubar, 1999; Sterman, 2000) including SUDs (Peniston, 1998; Peniston \& Kulkosky, 1989, 1990, 1991; Peniston, Marriman, Deming, \& Kulkosky, 1993) and continue to be a focal point for development of possible treatments for psychological disorders as well as discovery of functional processes (see current reviews by Hammond, 2006; Kaiser, 2006). A recent functional magnetic resonance imaging (fMRI) study reports neurofeedback techniques initiating blood oxygenated level dependent changes in the anterior cingulate cortex (ACC), caudate and substantia nigra in children with attention deficit hyperactivity disorder (Levesque, Beauregard, \& Mensour, 2006). In developing a protocol for the treatment of addictive disorders, it is paramount to have a sound theoretical foundation and evidence base. Neurofeedback protocols have been utilized for treatment of addictive disorders and are shown to be efficacious (see review by Sokhadze, Cannon, \& Trudeau, 2008). Despite numerous studies of neurofeedback and addiction, neurophysiological evidence of change has yet to be demonstrated, although neurofeedback research (Peniston \& Kulkosky, 1989, 1990, 1991) provides behavioral evidence for change, that is, personality testing, periods of abstinence, and other psychological and clinical outcomes.

Low-resolution electromagnetic tomographic (LORETA) neurofeedback (LNFB) provides the opportunity to train individuals to influence the electrical activity in regions not likely to be influenced by topographically specific EEG training. Based on prior studies (Cannon \& Lubar, in press; Cannon et al., 2007; Cannon et al., 2006) and two studies in review, we hypothesize that training $14-18 \mathrm{~Hz}$ activity in the right anterior cingulate gyrus (rACC) would directly influence activity in cortical and limbic regions of interest (ROI) shown to be significantly decreased or increased in recovering addicts as compared to nonclinical controls in eyesopened and assessment recordings (Cannon, Lubar, \& Baldwin, 2008). Hence data from this prior study influenced the a priori selection of ROI for this case study.

The participant in this study completed 25 sessions of LNFB training in the rACC with center Talairach coordinates at $(x=4$, $y=31, z=29)$. This article reports the data from the sessions in one participant including trend analysis, pre- and posttraining baseline comparisons and ROI analysis of the frequency specific relationships between the nine regions of interest as influenced by training in the rACC. As a result of our earlier studies successfully training individuals to increase activity in the cognitive division of the ACC (dorsal left BA 32/24; Cannon et al., 2007; Cannon et al., 2006), we hypothesize that the right division of the ACC (dorsal right BA 32/24) may involve 
more affective elements that would directly or indirectly influence regions shown to share connections with it, including the amygdala, hippocampus, anterior insula, and orbitofrontal regions. This region is often active in affect-related tasks, and cytoarchitecture studies show connections with thalamic nuclei, including the nucleus accumbens and hypothalamus (Bush, Luu, \& Posner, 2000; Devinsky, Morrell, \& Vogt, 1995). The ACC is demonstrated to be involved with regulation of autonomic, visceromotor, and endocrine systems and may be a region of integration between cognitive and affective processes. Lesions in the anterior ACC are shown to influence dysregulation of autonomic function, apathy, akinetic-mutism, alterations in consciousness, and personality, including lack of distress and affective lability (Bush et al., 2000; Davidson, Jackson, \& Kalin, 2000; Devinsky et al., 1995). The regions described in ACC reviews are shown active in studies relating to addiction, and many were identified in the aforementioned study in review; thus, Part 2 of our hypothesis is that there is a possible neural mechanism related to addiction and perception of self that will be influenced by LNFB training in the rACC.

Both case reports and research studies describe addicted individuals as passive with dependent strivings, emotionally immature, abounding with fears of responsibility or independent action, and ultimately infantile inadequate personalities (Coodley, 1961), as well as emotionally; socially; and, in some instances but not all, educationally underdeveloped (Meyerstein, 1964) and immature and regressive (Dorsey, 1961; Hill, 1962). Addicted individuals are reported to struggle with affirming positive thoughts of selfesteem and tendencies to undervalue themselves and be self-deprecating; they exhibit difficulty adjusting to others, and these tendencies are veiled by overt behavioral patterns, including physical or verbal abuse. Contradictory to what is often implied, these features may originate not from the consequences of substance abuse but from earlier periods in development (Vos, 1989). It is reasonable to infer that the ROI in this case study play a particular role in many of the abovementioned personality and psychological processes based on supporting imaging studies of fear, self-reference, decision making, motivation, reward, and face recognition, just to name a few (Blackwood et al., 2000; Cabeza et al., 2004; Fossati et al., 2003; Fossati et al., 2004; Grady \& Keightley, 2002; Jabbi, Swart, \& Keysers, 2007; Seger, Stone, \& Keenan, 2004; Simons, Davis, Gilbert, Frith, \& Burgess, 2006), hence we sought to demonstrate the efficacy of training low-beta power in the rACC and to examine the frequency specific effects in specific regions of the cortex.

\section{METHODS}

In this study we use the three-shell concentric spherical head model implementation made available from the Key Institute for Brain-Mind Research, Zurich, Switzerland. In this implementation, the current density is mapped for 2,394 voxels of dimension $7 \times 7 \times 7 \mathrm{~mm}$ covering the entire neocortex plus the ACC and hippocampus. In conventional neurofeedback, EEG activity is recorded at a particular scalp location. The physiological measurements are extrapolated from the signal and converted into auditory stimuli or visual objects that animatedly covary with the magnitude of a specified frequency or frequency band-pass region. Similarly, LNFB correlates the physiological signal with a continuous feedback signal; however, the physiological signal is defined as the current density in a specified region of training (ROT). This allows the continuous feedback signal to become a function of the intracranial current density and to covary with it (Congedo, 2006). The advantage over traditional neurofeedback is increased specificity of the training and the possibility to train activity in structures otherwise not identifiable by conventional EEG as originating in scalp measurements alone.

\section{Participant}

This study was accomplished with 1 female participant. She is 28 years of age 
and right-handed. She presents with 3 years of continuous abstinence maintained by active participation in 12-step programs. This participant reports first use of marijuana and LSD at age 13. She reports regular use of alcohol throughout adolescence and early adulthood. She began regular use of intravenous heroin at age 21 until age 25 . She reports family history of use and sexual, physical, and emotional abuse from age 3 to 12 . At 12 she was diagnosed with major depressive disorder. She reports two sexual assaults, one at 17 and one at 21 . She reports two previous suicide attempts; however, she relates these to drinking and drug use. She reports a history of antidepressant medications and expressed that these appeared to have no direct effects; otherwise, her medical history is unremarkable. At the onset and during the training she reports no overt depressive symptoms. She reports typical features associated with addicted individuals - struggling with positive selfimage and self-efficacy, inadequacy, racing thoughts that interfere with daily functioning and sleep patterns, negativism and pessimism, and being hypercritical of self and others. She read, signed, and agreed to an informed consent to protocol approved by the University of Tennessee Institutional Review Board. There is no reported history of head trauma, seizures, or recent drug or alcohol use.

\section{Procedures}

The participant was prepared for EEG recording using a measure of the distance between the nasion and inion to determine the appropriate cap size for recording (Electro-Cap International, Inc., Eaton, OH; see Blom \& Anneveldt, 1982). The head was measured and marked prior to each session to maintain consistency. The ears and forehead were cleaned for recording with a mild abrasive (NuPrep) gel to remove any oil and dirt from the skin. After fitting the caps, each electrode site was injected with electrogel and prepared so that impedances between individual electrodes and each ear were $<6 \mathrm{~K} \Omega$. The LNFB training was conducted using the 19-leads of the standard international 10/20 system with linked ears and ground reference. The electro-cap was referenced at FPz. The data were collected and stored utilizing the Deymed Truscan Acquisition system with a band-pass set at $0.5-64.0 \mathrm{~Hz}$ at a rate of 256 samples per second. We used standard $9 \mathrm{~mm}$ tin cup ear electrodes. Each session required approximately $60 \mathrm{~min}$ to complete. The frequencies analyzed are delta $(1.0-3.5 \mathrm{~Hz})$, theta $(3.5-$ $8.0 \mathrm{~Hz})$, alpha $1(8.0-10.0 \mathrm{~Hz})$, alpha 2 $(10.0-12.0 \mathrm{~Hz})$, beta $1(13-18 \mathrm{~Hz})$, beta 2 $(18-24 \mathrm{~Hz})$, and beta $3(24-32 \mathrm{~Hz})$.

\section{Neurofeedback Protocol}

Twenty-five LNFB training sessions, composed of pre- and postsession 3-min eyesclosed and eyes-opened baselines and four 5-min training rounds, were conducted three times per week. Following standard protocol (Cannon et al., 2007; Congedo, Lubar, \& Joffe, 2004), we designed to improve affective and executive processes by training this individual to increase $14-18 \mathrm{~Hz}$ (low-beta) power activity in a seven-voxel cluster of neurons in the right ACC with center coordinates at $x=4, y=31, z=29$. In a preliminary session, the participant was instructed to control tongue and eye movements, eye blinks, and muscle activity from forehead, neck, and jaws. This enabled her to minimize the production of extracranial artifacts (EMG, EOG, etc.) during the sessions. At the end of the preliminary session, the participant was informed of the inhibitory and reward aspects of the training. Standardized thresholds were then set and maintained based on prior research (Cannon et al., 2007; Cannon et al., 2006). One important addition to this particular protocol was concentration on minimizing the "therapist effect." This participant was exposed to the technician conducting the sessions only during the baseline recordings. During the actual training rounds there was a $45-\mathrm{sec}$ pause between rounds and the technician returned near the end of Round 4.

Following Congedo et al. (2004) the participant was provided visual and auditory feedback, and points were achieved when 
she was able to simultaneously maintain all conditions specified in Cannon et al. (2007). Maintaining all conditions for 0.75 sec achieved 1 point. The auditory stimuli provided positive reinforcement, a pleasant tone when the criteria were met. Similarly, the visual stimuli were activated when the criteria were being met (e.g., a car or a spaceship driving faster and straighter). Alternatively, a slower car, driving in the wrong lane or the spaceship flying slow and crooked, occurred when the criteria were not being met. The score for meeting the criteria was also seen by the participant in a small window of the game screen.

\section{Data Preprocessing}

In contrast with studies utilizing traditional neurofeedback, the whole-head EEG data were continuously stored during the sessions. All EEG data were processed with particular attention given to the frontal and temporal leads. All episodic eye blinks, eye movements, teeth clenching, jaw tension, body movements, and possible electrocardiogram were removed from the EEG stream. Fourier cross-spectral matrices were computed and averaged over $75 \%$ overlapping 4-sec artifact-free epochs, which resulted in one cross-spectral matrix for each participant and for each discrete frequency. These cross-spectral matrices constitute the input for LORETA estimation in the frequency domain. In this study, we focus on nine ROIs, of which one is the active ROT; the other eight, the secondary ROIs, have been found to be functionally associated to it. Table 1 lists the Talairach coordinates for the center voxel, the number of voxels composing the ROI, and its Brodmann area/ anatomical labeling.

\section{Data Analysis}

To appraise the linear increase of the ROIs with the rACC within the covariance structure, we extrapolated and averaged the values for the activity across the four rounds within each session. We then conducted analysis of variance (ANOVA) and regression
TABLE 1. Center Talairach coordinates for ROls, number of voxels comprising each ROI, Brodmann Area, and anatomical label.

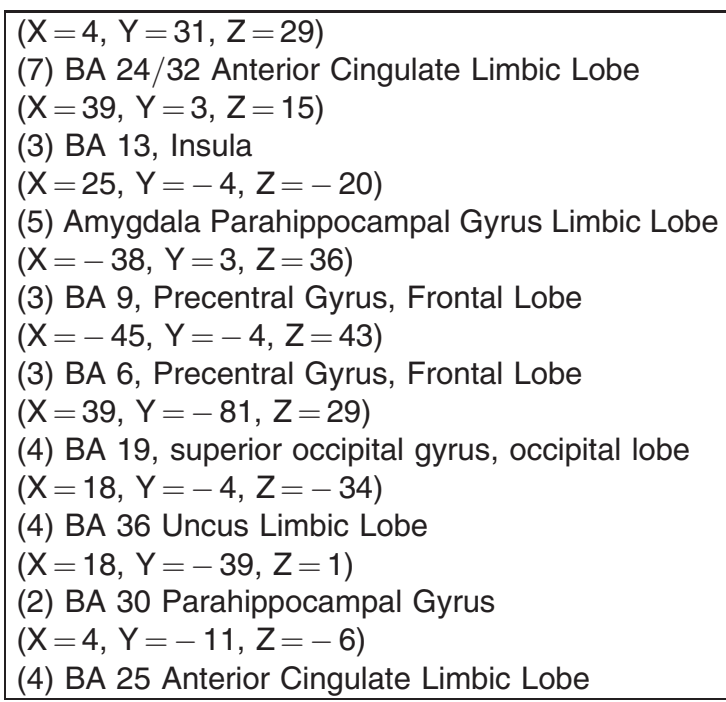

procedures evaluating the learning curves (i.e., the obtained values over sessions) for the ROT and ROIs utilizing PROC Mixed for complex linear mixed models in SAS 9.13 (Cannon et al., 2007; Schabenberger \& Pierce, 2002). In basic terms, an ANOVA model can be written as a linear model. The resulting equation predicts the response as a linear function of both parameters and design variables. Once models are expressed in the framework of linear models, hypothesis tests are expressed in expressions of a linear function of the parameters. Each fixed effect is a single variable or combination of variables (Fruend \& Littell, 2000; Neter, Kutner, Wasserman, \& Nachtsheim, 2005; Wackerly, Mendenhall, \& Scheaffer, 2002). This step was conceived to provide information regarding the degree to which training $14-18 \mathrm{~Hz}$ in the ROT produced effects for frequencies and increased or decreased current source density within the eight cortical regions (ROIs) and to differentiate functional activation patterns between rACC and the ROIs. We compared the ROT with the other ROIs for each of the EEG frequencies. The experiment-wise error rate was maintained at 0.01 using Tukey methodology (Cook \& Farewell, 1996). This 
method of analyzing neurofeedback has been published (Cannon et al., 2007), and other works are currently under review. We extrapolated the data from the regions of interest in Table 1 and compared them for significance. After averaging across the four rounds within each session for each ROI, we conducted a Pearson partial correlation analysis to assess the positive or negative relationship of the current density changes in the nine ROIs as a result of training 14 $18 \mathrm{~Hz}$ in right ACC. The partial correlation measures the strength of a relationship between two variables while controlling for the effects of other variables. The Pearson partial correlation between two variables, after controlling for other model variables is equivalent to the Pearson correlation between the residuals of the two variables after regression on the controlling variables. This stage was conceived to individuate those ROIs in which current density amplitude tends to increase (positive correlation) or decrease (negative correlation) with the rACC as a function of the neurofeedback learning process (i.e., strengthening of a network). We utilized the partial procedure to control for the possible effects of volume conduction as defined by Misulis (1993), that is, we assessed the correlation between each ROI and any other region controlling for the influence of the remaining seven regions. Threshold of significance for the correlation coefficients $r$ was set using the well known False Discovery Rate multiple testing procedure of Benjamini and Hochberg (1995, 1997). This procedure ensures that, on average, the proportion of falsely rejected hypotheses among the rejected ones is less than $q=.01$ for all tests.

\section{LORETA Voxel by Voxel Comparisons}

To assess the electrophysiological differences between this participant's pre- and post-eyes-opened baselines over the entire neo-cortex, we conducted all voxel-by-voxel $t$ tests setting the threshold to abs $(t)=4.0$ utilizing a multiple hypothesis testing procedure for the within-subjects experimental design. We performed this analysis utilizing log-transform, spatial normalization and $21.0 \mathrm{~mm}$ moving average. We maintained the family wise error rate with alpha set at 0.01 with 5,000 data randomizations.

\section{RESULTS}

\section{Trend Analysis in Specific ROIs}

We extrapolated and averaged current density for all training rounds for Sessions $1,5,10,15,20$, and 25, respectively. Figures 1 through 4 show the linear trends for the mean current density for the five regions of interest in the alpha 1, alpha 2 , beta, and training frequency (TF) as a result of training $14-18 \mathrm{~Hz}$ at the right ACC. From left to right and top to bottom the images show TF, alpha 1 , alpha 2 , and beta frequencies. On the $y$ axis is the average current density for the four rounds combined and on the $x$ axis are sessions. The mixed model results indicate that the learning curve for the trained frequency in the right ACC, although in the desired direction is not significant. This is not unexpected as we projected between 30 and 40 sessions would be necessary to facilitate a significant learning effect. There were no fixed effects produced by increase or decrease in the delta, alpha 1, beta 2 , or beta 3 frequencies at the right

FIGURE 1. Linear trend for the trained frequency over sessions for each region of interest; the graphs illustrate the mean current source density $(y)$ over sessions $(x)$. Note. 1 through 6 equate to Sessions 1 through 25, respectively. OFC = orbitofrontal cortex; AmC = amygdaloid complex; HIP = hippocampus; $\mathrm{OCC}=$ occipital lobe; $\mathrm{AC}=$ anterior cingulate.

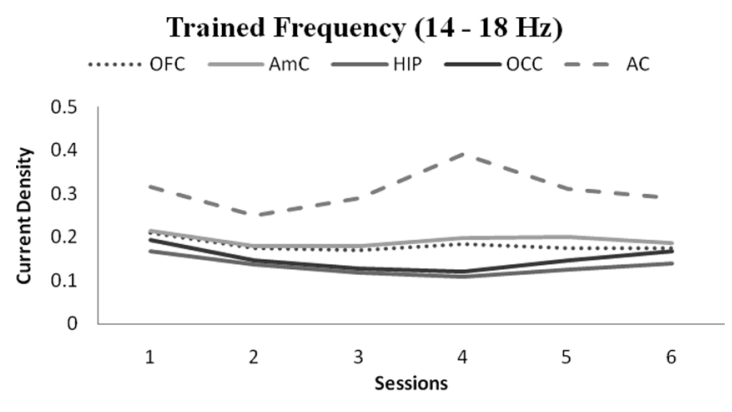


FIGURE 2. Linear trend for alpha 1 across sessions in each region of interest; the graphs illustrate the mean current source density $(y)$ over sessions $(x)$. Note. 1 through 6 equate to Session 1 through 25, respectively. $\mathrm{OFC}=$ orbitofrontal cortex; $\mathrm{AmC}=$ amygdaloid complex; HIP = hippocampus; $\mathrm{OCC}=$ occipital lobe; $\mathrm{AC}=$ anterior cingulate.

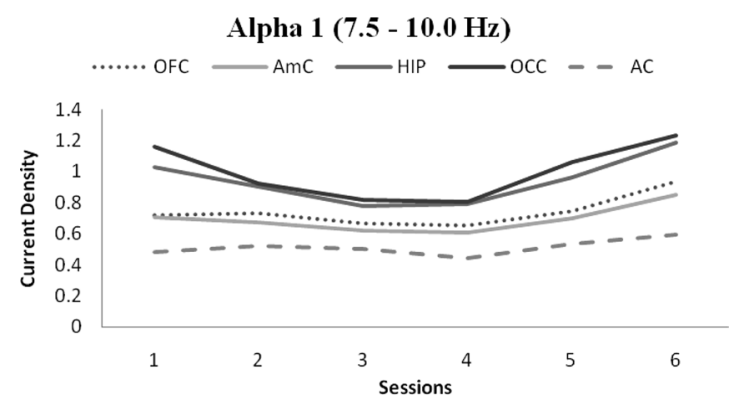

ACC. The type III test of fixed effects with 1, 7 degrees of freedom from the mixed model analysis indicate that training $14-18 \mathrm{~Hz}$ in the right ACC produces significant increase in BA 6, $F(8.44), p=.0228$, and BA 9, $F$ (9.40), $p=.0182$. Similarly, the increase of alpha 2 at the rACC produced frequency specific effects in the hippocampus, $F$ (9.16), $p=.0192$, and in the uncus, $F(8.61)$, $p=.0219$. The decrease of theta activity in the right ACC produced frequency specific effects in the hippocampus, $F(16.43)$, $p=.0049 ; \mathrm{BA} 19, F(9.54), p=.0176$; and the OFC, $F(7.22), p=.0312$.

FIGURE 3. Linear trend for alpha 2 across sessions in each region of interest; the graphs illustrate the mean current source density $(y)$ over sessions $(x)$. Note. 1 through 6 equate to Session 1 through 25, respectively. $\mathrm{OFC}=$ orbitofrontal cortex; $\mathrm{AmC}=$ amygdaloid complex; HIP = hippocampus; $\mathrm{OCC}=$ occipital lobe; $A C=$ anterior cingulate.

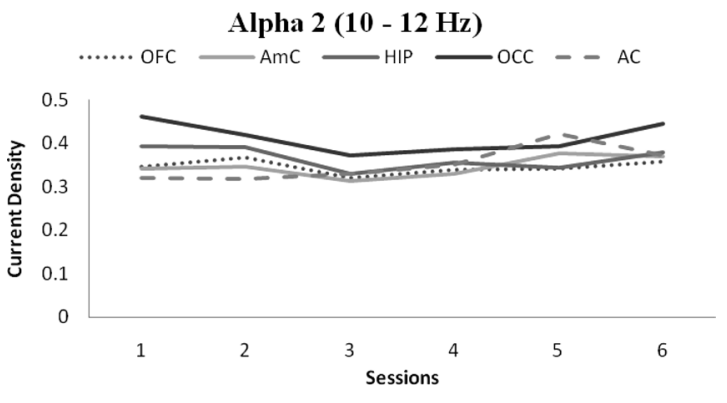

FIGURE 4. Linear trend for beta activity in each region of interest; the graphs illustrate the mean current source density $(y)$ over sessions $(x)$. Note. 1 through 6 equate to Session 1 through 25, respectively. $\mathrm{OFC}=$ orbitofrontal cortex; $\mathrm{AmC}=$ amygdaloid complex; HIP = hippocampus; OCC = occipital lobe; $\mathrm{AC}=$ anterior cingulate.

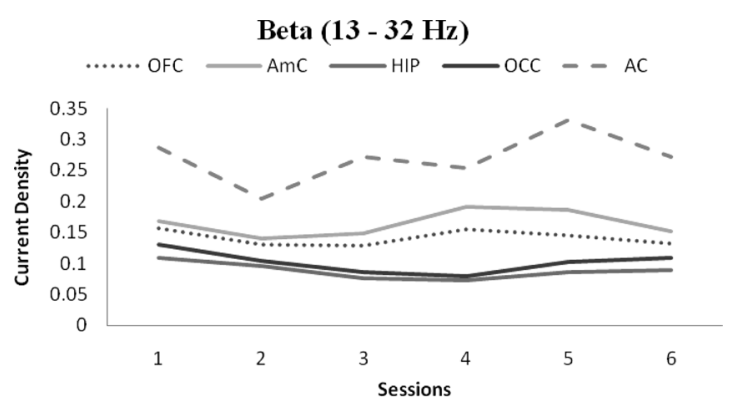

\section{Partial Correlations}

Table 2 shows the results for the Pearson partial correlation analysis for five specific regions of interest. In the table from top to bottom and left to right are the frequencies, rho, and probability of rho for (a) BA 19, (b) the hippocampus, (c) the amygdaloid complex, (d) BA 25, and (e) the rACC. The values shown in the table are those meeting the statistical criteria set with False Discovery Rate. The cluster of voxels within BA 19 share a significant association with the hippocampus in most frequencies and significant associations with the right ACC and hippocampus in the alpha 2 frequency. The alpha 2 frequency also shows both positive and negative associations with the left frontal regions which are in close proximity to one another. The cluster of voxels within the hippocampus shows positive associations with BA 19 and the OFC in most frequencies and similar positive and negative associations with the frontal regions as BA 19. The cluster of voxels within the amygdaloid complex shows the largest number of associations in the delta frequency with frontal and limbic regions. This cluster also shows a significant positive association with the insular cortex and a negative association with the right $\mathrm{ACC}$ in the theta frequency. Similarly, the AmC shares significant associations with the uncus in all frequencies and a positive 


\begin{tabular}{|c|c|c|}
\hline & 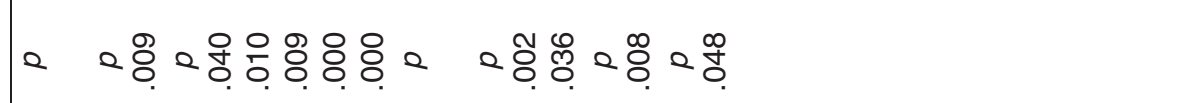 & \\
\hline 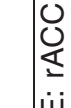 & 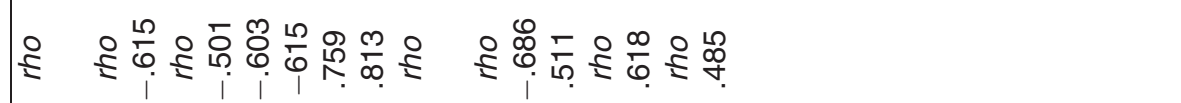 & $\bar{\Xi}$ \\
\hline & 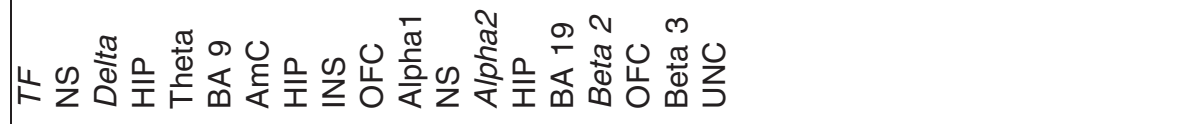 & \\
\hline & 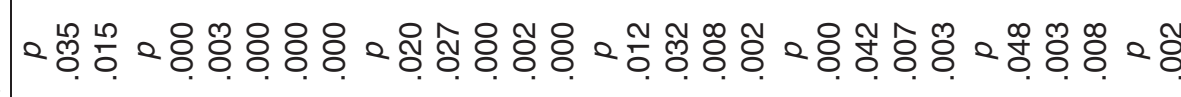 & ชั \\
\hline 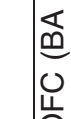 & 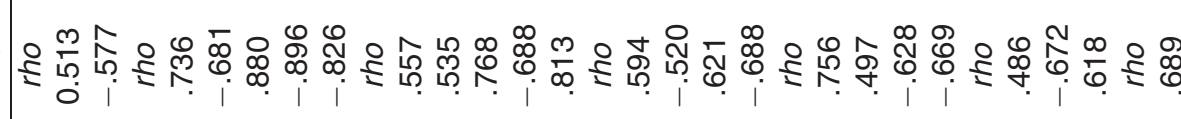 & $\stackrel{8}{:}$ \\
\hline$\ddot{a}$ & 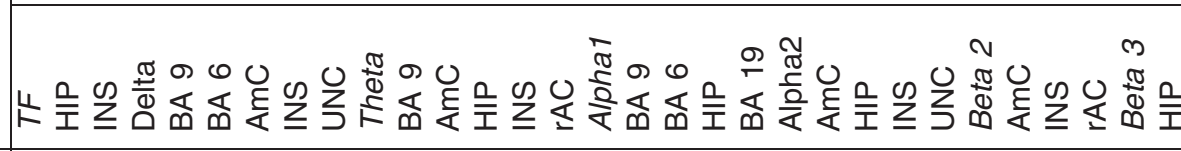 & $\frac{0}{1}$ \\
\hline 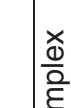 & 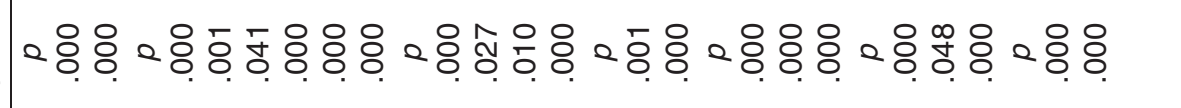 & \\
\hline$\frac{0}{\frac{0}{\pi}}$ & 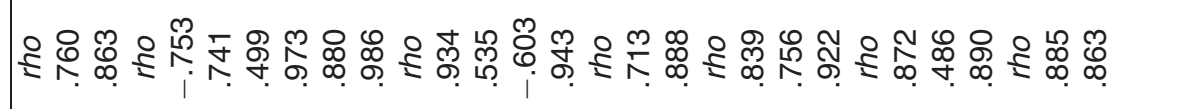 & \\
\hline 实 & 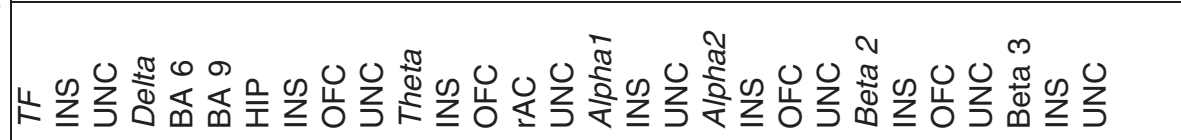 & \\
\hline$\underline{g}$ & 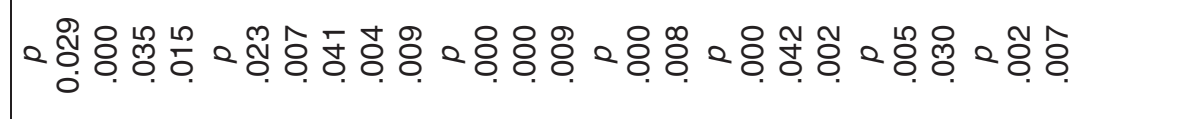 & \\
\hline 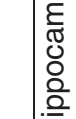 & 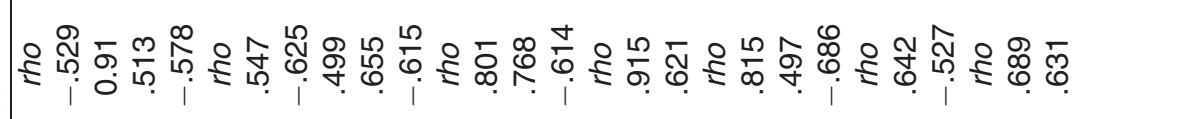 & \\
\hline$\ddot{\oplus}$ & 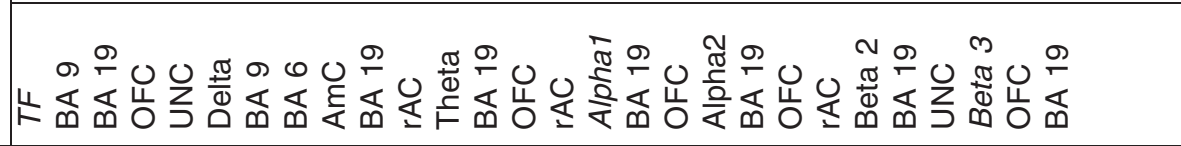 & \\
\hline$\Phi$ & 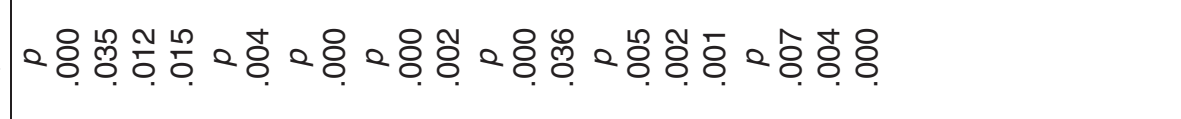 & \\
\hline$\frac{0}{9}$ & 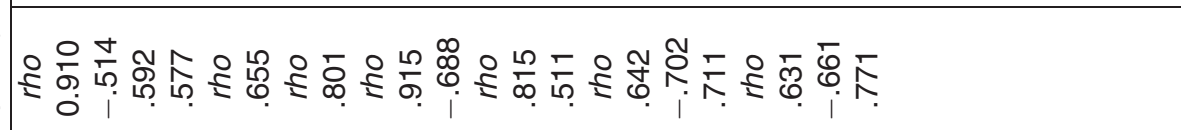 & \\
\hline$\ddot{\varangle}$ & 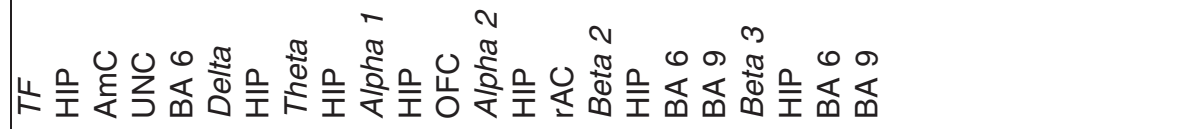 & \\
\hline
\end{tabular}


association with the hippocampus in only the delta frequency. The cluster of voxels within the OFC shows the greatest number of associations for all ROIs. It shares significant associations with the AmC in the delta, theta, alpha 2, and beta 2 frequencies. The OFC shares a negative association with the insular cortex in all frequencies. The cluster of voxels within the right ACC shows associations with regions similar to the linear mixed model effects. There are no associations with other regions in the delta, alpha 1 , or trained fre- quencies. The theta frequency shows positive associations with the insula and $\mathrm{OFC}$ and negative associations with the AmC, hippocampus, and BA 9. Of interest are the alpha 2 frequency and the apparent frequency specific negative association with the hippocampus and positive association with BA 19.

\section{sLORETA Estimations}

Figure 5 shows the results for the preposttraining baseline comparisons. In the

FIGURE 5. Participant pre-postsession eyes-opened baseline comparison from top to bottom: (A) theta, (B) alpha 1, (C) alpha 2, and (D) beta 1. Note. To the right of each image is the maximum and minimum reported by the sLORETA statistics.

\begin{tabular}{|c|c|c|}
\hline \multicolumn{2}{|l|}{ sLORETA Statistical Maps } & Maximum/Minimum \\
\hline 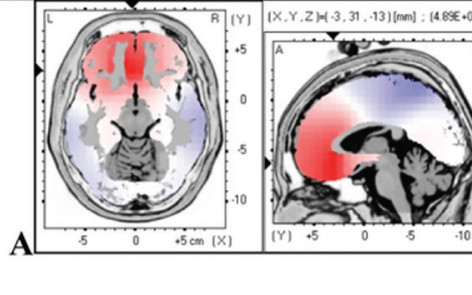 & 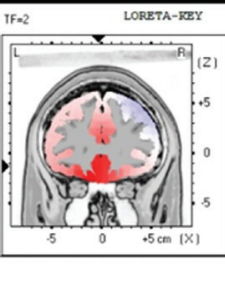 & $\begin{array}{l}(X=-10, Y=31, Z=-20) \\
\text { Brodmann area } 25 \\
\text { Medial Frontal Gyrus } \\
\text { Frontal Lobe } \\
(X=-31, Y=-46, Z=64) \\
\text { Brodmann area } 5 \\
\text { Postcentral Gyrus } \\
\text { Parietal Lobe }\end{array}$ \\
\hline 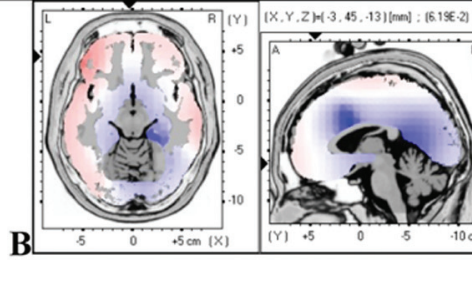 & 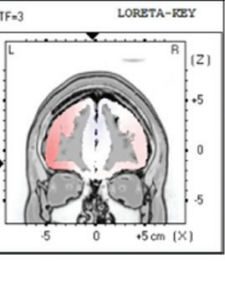 & $\begin{array}{l}(X=-52, Y=31, Z=-6) \\
\text { Brodmann area } 47 \\
\text { Inferior Frontal Gyrus } \\
\text { Frontal Lobe } \\
(X=-3, Y=10, Z=29) \\
\text { Brodmann area } 33 \\
\text { Anterior Cingulate } \\
\text { Limbic Lobe }\end{array}$ \\
\hline 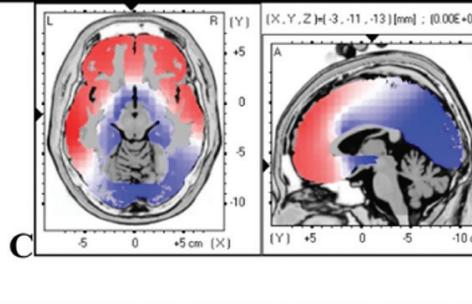 & $\int_{-5}^{2}$ & $\begin{array}{l}(\mathrm{X}=-66, \mathrm{Y}=-32, \mathrm{Z}=-20) \\
\text { Brodmann area } 21 \\
\text { Middle Temporal Gyrus } \\
\text { Temporal Lobe } \\
(\mathrm{X}=-3, \mathrm{Y}=-11, \mathrm{Z}=-6) \\
\text { Brodmann area } 25 \\
\text { Anterior Cingulate } \\
\text { Limbic Lobe }\end{array}$ \\
\hline 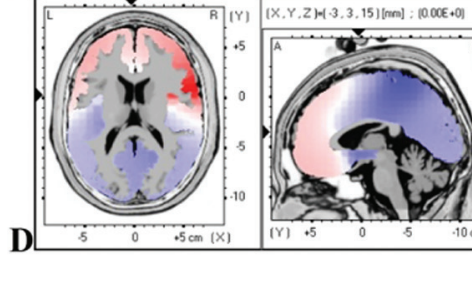 & 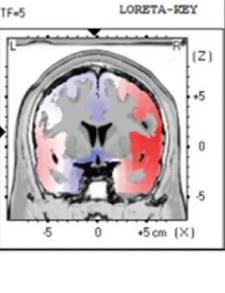 & $\begin{array}{l}(\mathrm{X}=53, \mathrm{Y}=10, \mathrm{Z}=1) \\
\text { Brodmann area } 22 \\
\text { Superior Temporal Gyrus } \\
\text { Temporal Lobe } \\
(\mathrm{X}=4, \mathrm{Y}=-39, \mathrm{Z}=71) \\
\text { Brodmann area } 4 \\
\text { Paracentral Lobule } \\
\text { Parietal Lobe }\end{array}$ \\
\hline
\end{tabular}


image from top to bottom are the theta, alpha 1, alpha 2, and beta 1 frequencies. The images from left to right are coronal, saggital, and axial views of the brain. The red in the image indicates regions of significant increased current density at .01 , and the blue indicates regions of significant decreased current density at .01 . To the right of each image is the maximum (top) and minimum (bottom) voxel estimated by sLORETA. The maximum increase in current density in the theta frequency is reported at BA 25 in medial frontal cortex and the maximum decrease is reported at BA 5 in the post central gyrus. The maximum increase in current density in the alpha 1 frequency is reported at BA 47 in the inferior frontal gyrus and the maximum decrease in current density is reported at BA 33 in the anterior cingulate. The maximum increase in current density in the alpha 2 frequency is reported at BA 21 in the middle temporal gyrus and the maximum decrease is reported at BA 25 in anterior cingulate. The maximum increase in current density in the beta 1 frequency is reported at BA 22 in superior temporal gyrus and the maximum decrease is reported at BA 4 in the paracentral lobule.

\section{DISCUSSION}

This is the first study of its kind to demonstrate relationships between limbic and cortical regions in specific frequencies as influenced by LORETA neurofeedback training in a recovering substance abuser; moreover, this type of analysis is not typically done to illustrate the frequency-specific neurophysiological changes that occur within the cortex as a result of the neurofeedback training process. The psychological processes discussed in this analysis of region and associations are limited by the scope of knowledge relating to neural networks and specific functioning of structures. In the following subsections we give a brief description of each region and attempt to provide a coherent interpretation and discussion. It is important to note that correlational and mixed model procedures evaluating the relationships between limbic and cortical regions are in the earliest of phases; however, this type of analysis affords the opportunity to understand how these regions interact with specificity. In brief, many of these relationships are counterintuitive to what we currently understand or expect from theoretical information. Similarly, it is important to consider that neurofeedback training in any one particular frequency does not produce effect for only that frequency; it produces effects in many different regions in many different frequencies. Thus our knowledge base of brain function can only be enhanced from such analytical procedures.

The regions of interest in this case study are shown to be active in tasks regarding social cognition, interpreting the mental states of others, emotion, memory, learning, decision making, reward, motivation and self reference, encoding, reality monitoring, suicide, empathetic and addictive processes (Abrahams et al., 2003; Adinoff, 2004; Alberto Tassinari et al. 2005; Bechara \& Damasio, 2002; Beer, Blakemore, Previc, \& Liotti, 2002; Berthoz, Armony, Blair, \& Dolan, 2002; Buss, Wolf, Witt, \& Hellhammer, 2004; Cannon, Lubar, Thornton, Wilson, \& Congedo, 2004; Critchley, 2005; du Boisgueheneuc et al., 2006; Fleck, Daselaar, Dobbins, \& Cabeza, 2006; Fossati et al., 2003; Goel, Grafman, Sadato, \& Hallett, 1995; Goldstein et al., 2007; Grady \& Keightley, 2002; Gusnard, 2005; Gusnard, Akbudak, Shulman, \& Raichle, 2001; Heiser, Iacoboni, Maeda, Marcus, \& Mazziotta, 2003; McNaughton et al., 1996; Stellar \& Corbett, 1989; Walton, Croxson, Behrens, Kennerley, \& Rushworth, 2007). One important concept regarding alpha activity in limbic regions as estimated by sLORETA and its relationship to SUD may be that these particular frequencies maintain a state of desynchronization within the individual, which in turn affects autonomic functioning, perceptual and cognitive processes, and numerous other personality and psychological factors. At the onset of LNFB training this subject did show excessive alpha activity in the OFC and limbic regions. Alcoholdependent individuals are reported to exhibit different synchronization of brain activity than light drinkers as reflected by differences 
in resting EEG coherence (Michael, Mirza, Mukundan, \& Channabasavanna, 1993; Winterer et al., 2003) and power (Bauer, 2001a, 2001b; Enoch, White, Harris, Rohrbaugh, \& Goldman, 2002; Rangaswamy et al., 2002; Rangaswamy et al., 2004; Saletu-Zyhlarz et al., 2004). Research has demonstrated the use of certain chemicals produces widespread alpha power increases in the cortex (Lukas, 1991, 1993, 1995), thereby, bringing the brain into synchrony, if only for a very short time. We believe this to be the euphoria addicts speak of so fondly and the reason for the difficulty in treating these disorders and high instance of relapse. It is reasonable to infer that instability of excitatory and inhibitory processes in limbic regions produce these patterns of activation, which become a relatively stable condition in SUD persons. This may reflect a tendency toward vacillation between past and future causing internal and external conflict, which leads to increased beta activity in the cortex resulting in increased anxiety, depression, panic, and attentional problems to which this participant affirms in her history. One possible area of interest is the degree that frequency specific activity in limbic regions influences dopamine production or restriction and what possible genetic factors are involved in perceptual processing of self in the substance abusing population. Levesque and colleagues (2004) conducted research exploring emotional regulation in children and found sad film excerpts elicits activity in similar regions as this research, namely, BA 9 and 10 in the left prefrontal cortex, the OFC and BA 11 and the right anterior cingulate as well as right BA 47 and these regions are shown to be influenced in this training. Studies have proposed that emotional self-regulation involves interactions of the midfrontal ACC with the amygdala (Posner \& Rothbart, 1998) and PFC damage in adults resembles normal reactions in children (Fox, Henderson, \& Marshall, 2001). Increased dopamine levels in limbic regions (Blum et al., 2007; Köhnke et al., 2003) are shown to result from ethanol use, and it is posited that individuals having low levels of a certain alleles may have low levels of dopamine in limbic regions thereby increasing the likelihood of developing an SUD and may also influence behavioral, social, and interpersonal difficulties exhibited by addicted persons even after years of abstinence. It is important to consider historical information the neuronal consequences of abuse, neglect, and inadequacy (real or imagined) and their influence in developmental neuronal processes. Alpha activity in limbic regions may be an area of vital importance to the study and treatment of SUD.

LORETA neurofeedback training in the rACC in this participant does appear to influence frequency specific activity increase and decrease in limbic and cortical regions of interest at significant levels. Although the increase in the trained frequency at the rACC did not meet statistical criteria, the influence of the training in other regions is significant. Figures 1 through 4 show the mean current density increase over sessions. These figures show the effects of training $14-18 \mathrm{~Hz}$ in $\mathrm{rACC}$ in five regions in alpha 1, alpha 2, TF, and beta frequency domains for five regions of interest across sessions. An interesting effect is the increased alpha activity in BA 19-this region in the left hemisphere is approximate to the $\mathrm{O} 1$ training by Peniston and colleagues (Peniston, 1998; Peniston \& Kulkosky, 1989, 1990, 1991). The mixed model and partial procedures suggest that this region and the hippocampus are intimately related. The meaning of this relationship and many others elucidated in this study are unknown at this time. The mixed model results indicate that training $14-18 \mathrm{~Hz}$ in the rACC produces direct effects in the left lateral prefrontal cortex (BA 6 and 9). Similarly, increased alpha 2 in the rACC produced frequency specific effects in the right hippocampus and uncus, whereas the decrease of theta in rACC produced significant effects in the hippocampus, BA 19, and OFC. These data may offer insight into the neural substrates of addiction as well as perceptual processes involving the self. One of the major differences occur in the alpha frequency, in prebaseline measures this participant showed excess alpha in limbic regions which at the end of training appears to be more dispersed to temporal 
areas. These mechanisms may possibly be influenced by single electrode training in occipital regions $(\mathrm{O} 1$, or $\mathrm{O} 2)$ or multisite (LCC) training; however, this cannot be asserted due to lack of neurophysiological evidence (e.g., LORETA, fMRI, PET) produced in such studies. Similarly, it may be posited that the changes that occurred in this participant can be attributed to habituation to the visual stimuli; however, our prior studies in the left ACC showed similar activation patterns found in real-time fMRI selfregulation training (Wesikopf et al., 2003, 2004). Similarly, the relationship between these regions as influenced by LNFB may in fact be an anomaly specific to this individual; however, our prior research indicates a similar pattern in a group of recovering addicts. The participant reports an improved sense of well-being, decreased anxiety, and stress. Of note are depressive features in her EEG at the end of training and increased alpha activity appearing specific to the LPFC, which may be an underlying antecedent to this individual's addictive disorder.

We can conclude that LNFB training in the rACC does produce changes within neural networks in regions influencing emotional regulation, personality, memory, and self-perceptual and inhibitory processing in a positive manner. This type of self-regulation in conjunction with current treatment models may offer an evidence-based procedure that will positively influence behavior and decrease the likelihood of recidivism in SUD. Larger studies are needed and planned to confirm the changes described in this article. Two important considerations for this method of treatment reported by the participant are (a) she did not report the calmness and sense of well being reported by Peniston; instead, between sessions 12 and 19 she reported "feeling more insane than she ever had, but it was manageable," which is possibly attributed to the changes occurring within limbic and anterior cingulate regions, and (b) because this participant had been abstinent for a significant period, those in early recovery may require a more controlled environment during these early sessions. Similarly, it may also be imperative to advise clients to avoid any novel reward-evoking behaviors during the treatment as we are dealing with regions susceptible to habituation. We plan to conduct a larger study to determine the prominence of this circuit and the ability to influence it using LNFB and to validate the effects with pre- and post-fMRI. We do expect significant learning to require more than 30 sessions; however, we do project that changes in these specific regions will afford the individual the opportunity to change negative perceptions of self and self-in-experience that continue to produce deleterious behaviors despite significant periods of abstinence. This case study may reflect the importance and necessity of developing integrative models for concomitantly treating the neuronal processes involved in the negative perceptions of self and self-in-experience and the cognitive and affective consequences associated with such negative thought processes.

\section{REFERENCES}

Abrahams, S., Goldstein, H., Simmons, A., Brammer, M., Williams, S., Giampietro, V. P., et al. (2003). Functional magnetic resonance imaging of verbal fluency and confrontation naming using compressed image acquisition to permit overt responses. Human Brain Mapping, 20, 29-40.

Adinoff, B. (2004). Neurobiologic processes in drug reward and addiction. Harvard Review of Psychiatry, 12, 305-320.

Alberto Tassinari, C., Tassi, L., Calandra-Buonaura, G., Stanzini-Maserati, M., Fini, N., Pizza, F., et al. (2005). Biting behavior, aggression, and seizures. Epilepsia, 46, 654-663.

Bauer, L. O. (2001a). CNS recovery from cocaine, cocaine and alcohol, or opioid dependence: A P300 study. Clinical Neurophysiology, 112, 1508-1515.

Bauer, L. O. (2001b). Predicting relapse to alcohol and drug abuse via quantitative electroencephalography. Neuropsychopharmacology, 25, 332-333.

Bechara, A., \& Damasio, H. (2002). Decision-making and addiction (part I): Impaired activation of somatic states in substance dependent individuals when pondering decisions with negative future consequences. Neuropsychologia, 40, 1675-1689.

Beer, J., Blakemore, C., Previc, F., \& Liotti, M. (2002). Areas of the human brain activated by ambient visual motion, indicating three kinds of self-movement. Experimental Brain Research. Experimentelle Hirnforschung. Experimentation Cerebrale, 143, 78-88. 
Benjamini, Y. \& Hochberg, Y. (1995). Controlling the False Discovery Rate - A practical and powerful approach to multiple testing. Journal of the Royal Statistical Society Series B Methodological, 57, 289-300.

Benjamini, Y., \& Hochberg, Y. (1997). Multiple hypotheses testing with weights. Scandinavian Journal of Statistics, 24, 407-418.

Berthoz, S., Armony, J., Blair, R., \& Dolan, R. (2002). An fMRI study of intentional and unintentional (embarrassing) violations of social norms. Brain, 125(Pt. 8), 1696-1708.

Blackwood, N. J., Howard, R., ffytche, D., Simmons, A., Bentall, R., \& Murray, R. (2000). Imaging attentional and attributional bias: An fMRI approach to the paranoid delusion. Psychological Medicine, 30, 873-883.

Blom, J. L., \& Anneveldt, M. (1982). An electrode cap tested. Electroencephalography and Clinical Neurophysiology, 54, 591-594.

Blum, K., Chen, T., Meshkin, B., Waite, R., Downs, B., Blum, S., et al. (2007). Manipulation of catechol-Omethyl-transferase (COMT) activity to influence the attenuation of substance seeking behavior, a subtype of Reward Deficiency Syndrome (RDS), is dependent upon gene polymorphisms: a hypothesis. Medical Hypotheses, 69, 1054-1060.

Bush, G., Luu, P., \& Posner, M. I. (2000). Cognitive and emotional influences in anterior cingulate cortex. Trends in Cognitive Sciences, 4, 215-222.

Buss, C., Wolf, O., Witt, J., \& Hellhammer, D. (2004). Autobiographic memory impairment following acute cortisol administration. Psychoneuroendocrinology, 29, 1093-1096.

Cabeza, R., Prince, S., Daselaar, S., Greenberg, D., Budde, M., Dolcos, F., et al. (2004). Brain activity during episodic retrieval of autobiographical and laboratory events: An fMRI study using a novel photo paradigm. Journal of Cognitive Neuroscience, 16, 1583-1594.

Cannon, R., \& Lubar, J. (in press). EEG spectral power and coherence: Differentiating effects of Spatial-Specific Neuro-Operant Learning (SSNOL) utilizing LORETA Neurofeedback training in the anterior cingulate and bilateral dorsolateral prefrontal cortices. Journal of Neurotherapy.

Cannon, R., Lubar, J., \& Baldwin, D. (2008) Selfperception and experiential schemata in the addicted brain. Appl Psychophysiol Biofeedback, 33(4): doi: 10.1007/s10484-008-9067-9.

Cannon, R., Lubar, J., Congedo, M., Thornton, K., Hutchens, T., \& Towler, K. (2007). The effects of Neurofeedback in the cognitive division of the anterior cingulate gyrus. The International Journal of Neuroscience, 117, 337-357.

Cannon, R., Lubar, J., Gerke, A., Thornton, K., Hutchens, T., \& McCammon, V. (2006).
Topographical coherence and absolute power changes resulting from LORETA Neurofeedback in the anterior cingulate gyrus. Journal of Neurotherapy, $10,5-31$.

Cannon, R., Lubar, J., Thornton, K., Wilson, S., \& Congedo, M. (2004). Limbic beta activation and LORETA: Can hippocampal and related limbic activity be recorded and changes visualized in an affective memory condition?. Journal of Neurotherapy, 8, 5-24.

Congedo, M. (2006). Subspace projection filters for real-time brain electromagnetic imaging. IEEE Transactions on Bio-Medical Engineering, 53, 1624-1634.

Congedo, M., Lubar, J., \& Joffe, D. (2004). Low-resolution electromagnetic tomography neurofeedback. IEEE Transactions on Neuronal Systems and Rehabilitation Engineering, 12, 387-397.

Coodley, A. E. (1961). Current aspects of delinquency and addiction. General Psychiatry, 4, 124-132, 646.

Cook, R., \& Farewell, V. T. (1996). Multiplicity considerations in the design and analysis of clinical trials. Journal of the Royal Statistical Society. Series A (General), 159, 93-110.

Critchley, H. D. (2005). Neural mechanisms of autonomic, affective, and cognitive integration. The Journal of Comparative Neurology, 493, 154-166.

Davidson, R., Jackson, D., \& Kalin, N. (2000). Emotion, plasticity, context, and regulation: Perspectives from affective neuroscience. Psychological Bulletin, 126, 890.

Devinsky, O., Morrell, M., \& Vogt, B. (1995). Review article: Contributions of anterior cingulate cortex to behaviour. Brain, 118, 279-306.

Dorsey, J. M. (1961). Narcotics addiction in our community primarily an educational problem. Journal-Michigan State Medical Society, 60, 621-629.

du Boisgueheneuc, F., Levy, R., Volle, E., Seassau, M., Duffau, H., Kinkingnehun, S., et al. (2006). Functions of the left superior frontal gyrus in humans: A lesion study. Brain, 129, 3315-3328.

Enoch, M. A., White, K. V., Harris, C. R., Rohrbaugh, J., \& Goldman, D. (2002). The relationship between two intermediate phenotypes for alcoholism: Low voltage alpha EEG and low P300 ERP amplitude. Journal of Studies on Alcohol, 63, 509-517.

Fleck, M., Daselaar, S., Dobbins, I., \& Cabeza, R. (2006). Role of prefrontal and anterior cingulate regions in decision-making processes shared by memory and nonmemory tasks. Cerebral Cortex, 16, 1623-1630.

Fossati, P., Hevenor, S., Graham, S., Grady, C., Keightley, M., Craik, F., et al. (2003). In search of the emotional self: An FMRI study using 
positive and negative emotional words. The American Journal of Psychiatry, 160(11), 1938-1945.

Fossati, P., Hevenor, S., Lepage, M., Graham, S., Grady, C., Keightley, M., et al. (2004). Distributed self in episodic memory: neural correlates of successful retrieval of self-encoded positive and negative personality traits. NeuroImage, 22, 1596-1604.

Fox, N. A., Henderson, H. A., \& Marshall, P. J. (2001) The biology of temperament: an integrative approach. In C. A. Nelson \& M. Luciana (Eds.), Handbook of developmental cognitive neuroscience (pp. 631-645. Cambridge, MA: MIT Press.

Fruend, R., \& Littell, R. (2000). SAS system for regression (3rd ed., pp. 157-185). New York: Wiley and Sons.

Goel, V., Grafman, J., Sadato, N., \& Hallett, M. (1995). Modeling other minds. Neuroreport, 6(13), 1741-1746.

Goldstein, R., Tomasi, D., Alia-Klein, N., Cottone, L., Zhang, L., Telang, F., et al. (2007). Subjective sensitivity to monetary gradients is associated with frontolimbic activation to reward in cocaine abusers. Drug and Alcohol Dependence, 87(2-3), 233-240.

Grady, C., \& Keightley, M. (2002). Studies of altered social cognition in neuropsychiatric disorders using functional neuroimaging. Canadian Journal of Psychiatry, 47, 327-336.

Gusnard, D. A. (2005). Being a self: Considerations from functional imaging. Consciousness and Cognition, 14, 679.

Gusnard, D. A., Akbudak, E., Shulman, G. L., \& Raichle, M. E. (2001). Medial prefrontal cortex and self-referential mental activity: Relation to a default mode of brain function. Proceedings of the National Academy of Sciences of the United States of America, 98, 4259-4264.

Hammond, D. C. (2006). What is neurofeedback? Journal of Neurotherapy, 10, 25-36.

Heiser, M., Iacoboni, M., Maeda, F., Marcus, J., \& Mazziotta, J. (2003). The essential role of Broca's area in imitation. The European Journal of Neuroscience, 17, 1123-1128.

Hill, H. E. (1962). The social deviant and initial addiction to narcotics and alcohol. Quarterly Journal of Studies on Alcohol, 23, 562-582.

Jabbi, M., Swart, M., \& Keysers, C. (2007). Empathy for positive and negative emotions in the gustatory cortex. NeuroImage, 34, 1744-1753.

Kaiser, D. A. (2006). What is quantitative EEG? Journal of Neurotherapy, 10, 37-52.

Köhnke, M. D., Wiatr, G., Kolb, W., Kohnke, A., Schick, S., Lutz, U., et al. (2003). Plasma homovanillic acid: A significant association with alcoholism is independent of a functional polymorphism of the human catechol-o-methyltransferase gene. Neuropsychopharmacology, 28, 1004-1010.
Levesque, J., Beauregard, M., \& Mensour, B. (2006). Effect of neurofeedback training on the neural substrates of selective attention in children with attention-deficit/hyperactivity disorder: A functional magnetic resonance imaging study. Neuroscience Letters, 394, 216-221.

Levesque, J., Joanette, Y., Mensour, B., Beaudoin, G., Leroux, J., Bourgouin, P., et al. (2004). Neural basis of emotional self-regulation in childhood. Neuroscience, 129, 361-369.

Lubar, J. F. (1997). Neocortical dynamics: Implications for understanding the role of neurofeedback and related techniques for the enhancement of attention. Applied Psychophysiology and Biofeedback, 22, 111-126.

Lubar, J. F., \& Lubar, J. (1999). Neurofeedback assessment and treatment for attention deficit/ hyperactivity disorders. In A. Abarbanel \& J. R. Evans (Eds.), Introduction to quantitative EEG and neurofeedback (pp. 103-143. San Diego, CA: Academic Press.

Lukas, S. E. (1991). Topographic mapping during cocaine-induced intoxication and self-administration. In G. Racagni, N. Brunello, \& T. Fukuda (Eds.), Biological psychiatry (Vol. 2, pp. 25-29). New York: Elsevier Science.

Lukas, S. E. (1993). Advanced electrophysiological imaging techniques for studying drug effects. In E. D. London (Ed.), Imaging drug action in the brain (pp. 389-404. Boca Raton, FL: CRC Press.

Lukas, S. E., Mendelson, J. H., \& Benedikt, R. (1995). Electroencephalographic correlates of marihuanainduced euphoria. Drug and Alcohol Dependence, 37, 131-140.

McNaughton, B., Barnes, C., Gerrard, J., Gothard, K., Jung, M., Knierim, H., et al. (1996). Deciphering the hippocampal polyglot: The hippocampus as a path integration system. The Journal of Experimental Biology, 199(Pt. 1), 173-185.

Meyerstein, A. N. (1964). Drug addiction: A review. The Journal of School Health, 34, 77-87.

Michael, A., Mirza, K. A., Mukundan, C., \& Channabasavanna, S. M. (1993). Interhemispheric electroencephalographic coherence as a biological marker in alcoholism. Acta Psychiatrica Scandinavica, 87, 213-217.

Misulis, K. (1993). Essentials of clinical neurophysiology (pp. 6-19). Boston: Butterworth-Heinemann.

Nestler, E. J. (2005). The neurobiology of cocaine addiction. Sci Pract Perspect, 3(1), 4-10.

Neter, J., Kutner, M., Wasserman, W., \& Nachtsheim, C. (1996). Applied linear statistical models (4th ed.). New York: McGraw Hill/Irwin.

Peniston, E. G. (1998). Comments by Peniston. Applied Psychophysiology and Biofeedback, 23, 273-275. 
Peniston, E. G., \& Kulkosky, P. J. (1989). Alpha-theta brainwave training and beta endorphin levels in alcoholics. Alcoholism, Clinical and Experimental Research, 13, 271-279.

Peniston, E. G., \& Kulkosky, P. J. (1990). Alcoholic personality and alpha-theta brainwave training. Medical Psychotherapy, 2, 37-55.

Peniston, E. G., \& Kulkosky, P. G. (1991). Alphatheta brain wave neurofeedback for Vietnam veterans with combat related post traumatic stress disorder. Medical Psychotherapy, 4, 1-14.

Peniston, E. G., Marriman, D. A., Deming, W. A., \& Kulkosky, P. G. (1993). EEG alpha-theta brain wave synchronization in Vietnam theater veterans with combat related post traumatic stress disorder and alcohol abuse. Medical Advances in Medical Psychotherapy, 6, 37-50.

Posner, M. I., \& Rothbart, M. K. (1998). Attention, self-regulation and consciousness. Philosophical Transactions of the Royal Society of London. Series B, Biological Sciences, 353, 1915-1927.

Rangaswamy, M., Porjesz, B., Chorlian, D., Wang, K., Jones, K., Bauer, L. O., et al. (2002). Beta power in the EEG of alcoholics. Biological Psychiatry, 52, 831-842.

Rangaswamy, M., Porjesz, B., Chorlian, D., Wang, K., Jones, K., Kuperman, S., et al. (2004). Resting EEG in offspring of male alcoholics: Beta frequencies. International Journal of Psychophysiology, 51, 239-251.

Saletu-Zyhlarz, G. M., Arnold, O., Anderer, P., Oberndorfer, S., Walter, H., Lesch, O. M., et al. (2004). Differences in brain function between relapsing and abstaining alcohol-dependent patients, evaluated by EEG mapping. Alcohol and Alcoholism (Oxford, Oxfordshire), 39, 233-240.

Schabenberger, O., \& Pierce, F. J. (2002) Contemporary statistical models for the plant and soil sciences. Boca Raton, FL: CRC Press.

Seger, C., Stone, M., \& Keenan, J. (2004). Cortical Activations during judgments about the self and another person. Neuropsychologia, 42, 1168-1177.

Simons, J., Davis, S., Gilbert, S., Frith, C., \& Burgess, P. (2006). Discriminating imagined from perceived information engages brain areas implicated in schizophrenia. NeuroImage, 32, 696-703.

Sokhadze, T., Cannon, R., \& Trudeau, D. (2008). (White Paper) EEG biofeedback as a treatment for substance use disorders: Review, rating of efficacy and recommendations for further research. Journal of Applied Psychophysiology and Biofeedback, 33, 1-28.

Stellar, J., \& Corbett, D. (1989). Regional neuroleptic microinjections indicate a role for nucleus accumbens in lateral hypothalamic self-stimulation reward. Brain Research, 477, 126-143.

Sterman, B. (2000). EEG markers for attention deficit disorder: Pharmacological and neurofeedback applications. Child Study Journal, 30, 1-23.

Vos, H. (1989). Denial of the inner reality: Observations on drug abuse and addiction based on psychotherapies after treatment in a therapeutic community in the Netherlands. Journal of Substance Abuse Treatment, 6, 193-199.

Wackerly, D., Mendenhall, W., \& Scheaffer, R. (2002). Mathematical statistics with applications (6th ed., pp. 629-672). Pacific Grove, CA: Duxbury.

Walton, M., Croxson, P., Behrens, T., Kennerley, S., \& Rushworth, M. (2007). Adaptive decision making and value in the anterior cingulate cortex. NeuroImage, 36(Suppl. 2), T142-T154.

Weiskopf, N., Scharnowski, F., Veit, R., Goebel, R., Birbaumer, N., \& Mathiak, K. (2004). Selfregulation of local brain activity using real-time functional magnetic resonance imaging (fMRI). J Physiol Paris, 98(4-6), 357-373.

Weiskopf, N., Veit, R., Erb, M., Mathiak, K., Grodd, W., Geobel, R., et al. (2003). Physiological self-regulation of regional brain activity using real-time functional magnetic resonance imaging (fMRI): methodology and exemplary data. NeuroImage, 19, 577-586.

Winterer, G., Enoch, M. A., White, K., Saylan, M., Coppola, R., \& Goldman, D. (2003). EEG phenotype in alcoholism: Increased coherence in the depressive subtype. Acta Psychiatrica Scandinavica, 108, 51-60. 